\title{
Incidence of Bactobilia Increases over Time after Endoscopic Sphincterotomy
}

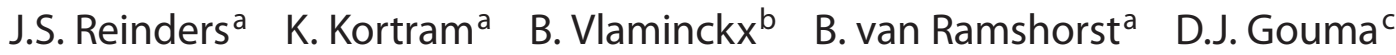 \\ D. Boerma ${ }^{a}$ \\ Departments of a Surgery and ${ }^{\mathrm{b}}$ Medical Microbiology and Immunology, St. Antonius Hospital, Nieuwegein, and \\ 'Department of Surgery, Academic Medical Center, Amsterdam, The Netherlands
}

\section{Key Words}

Choledochocystolithiasis - Endoscopic sphincterotomy • Laparoscopic cholecystectomy $\cdot$ Bile culture $\cdot$ Bactobilia

\begin{abstract}
Patients with choledochocystolithiasis are usually treated by endoscopic retrograde cholangiography with endoscopic sphincterotomy (ES) followed by laparoscopic cholecystectomy (LC). LC after ES is more difficult than in uncomplicated gallstone disease, possibly due to bacterial colonization of the common bile duct. The goal of this study was to evaluate if bactobilia influences the peri- and postoperative outcomes. Data were obtained from a randomized trial on the timing of LC after ES. Ninety-six patients were randomized after ES to LC either within $72 \mathrm{~h}$ (early LC [ELC]) or in 6-8 weeks (delayed LC [DLC]). In 64 of 96 patients bile samples were obtained peroperatively. The overall prevalence of bactobilia was $62.5 \%[40 / 64 ; 50 \%$ of ELC patients $(n=13)$ vs. $71.1 \%$ in the DLC group ( $n=27) ; p=0.088$ ]. Age and group (i.e. ELC/DLC) were independent and significant predictors for the presence of bactobilia. The presence of bactobilia did not influence operating time and difficulty or conversion rate. Patients with bactobilia developed more biliary events in the period between ES and LC (44 vs. 28\%). After ES for
\end{abstract}

choledochocystolithiasis, $62.5 \%$ of patients have bactobilia at the time of surgery. The prevalence of bactobilia increases with age and time. Patients with bactobilia tend to develop more biliary-related complications awaiting surgery.

Copyright $\odot 2011$ S. Karger AG, Basel

\section{Introduction}

For patients with combined choledochocystolithiasis endoscopic retrograde cholangiography (ERCP) with endoscopic sphincterotomy (ES) followed by laparoscopic cholecystectomy (LC) is still the most common treatment. A Cochrane review concluded that patients benefit from a prophylactic LC within 6 weeks in comparison to deferral of the LC [1]. Patients in the LC group had significantly better primary outcome (mortality) and secondary outcomes (biliary complications, subsequent ERCP or cholecystectomy). Therefore, the authors' conclusion was that an LC should be offered to anyone who is deemed fit for surgery. In a recent randomized clinical trial, we showed that performing LC within $72 \mathrm{~h}$ after ES is safe and prevents recurrent biliary complications in patients awaiting LC after ES within 6-8 weeks [2]. A similar randomized clinical trial by Salman et al. [3] in which

\section{KARGER \\ Fax +41 613061234 E-Mail karger@karger.ch} www.karger.com

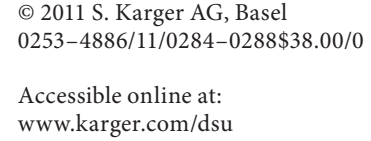

D. Boerma

Department of Surgery, St. Antonius Hospital

Koekoekslaan 1, PO Box 2500

NL-3430 EM Nieuwegein (The Netherlands)

Tel. +31 30609 9111, E-Mail djamilaboerma@ hotmail.com 
they compared LC after ES for choledochocystolithiasis within $72 \mathrm{~h}$ versus after $72 \mathrm{~h}$ showed a significant lower conversion rate, a shorter median operating time and shorter median postoperative hospital stay in the group which received their LC within $72 \mathrm{~h}$.

Multiple studies demonstrate that LC after ES is more difficult than in uncomplicated gallstone disease and has a higher conversion rate $[1,4,5]$. A probable explanation for this phenomenon is the destruction of the sphincter of Oddi. This may lead to bacterial colonization of the common bile duct, inflammation and scarring of the hepatoduodenal ligament $[3,5-9]$. The goal of this study was to assess the rate of bactobilia in patients undergoing LC after previous ES for bile duct stones, and to determine its effect on the outcome of LC and postoperative morbidity.

\section{Patients and Methods}

Patients were recruited from a multicenter randomized clinical trial studying the effects of the timing of LC after ES for combined choledochocystolithiasis [2]. In this trial, 96 patients were randomly allocated to LC either within $72 \mathrm{~h}$ after ES (early LC [ELC] group) or 6-8 weeks after ES (delayed LC [DLC] group). All patients were 18 years and older and had a ASA classification under IV.

Patients undergoing ES were not routinely administered antibiotics during or following the procedure.

The LC was done by a standard four-trocar technique. During the LC, a bile sample was collected under aseptic conditions.

Bile samples were routinely cultured on aerobic (blood, chocolate and MacConkey agar) and anaerobic media for 5 days at $37^{\circ} \mathrm{C}$ and were evaluated for aerobic and anaerobic bacteria, as well as growth of fungi and yeast.

Duration and difficulty of surgeries, conversion rate, postoperative complications, hospital stay and recurrent biliary events (while waiting for LC) were recorded. The difficulty of LC was scored by the most experienced surgeon in the operating team on a $0-10$ scale, with ' 0 ' (zero) being very easy and ' 10 ' very difficult. Operating time was defined as 'time between first incision and placement of last suture'. The surgeons' experience was recorded. If conversion occurred, the surgeon had to report the reason for conversion. Follow-up consisted of a visit to the outpatient clinic 2 weeks after LC and telephone interviews after 6 weeks and 6 months.

Statistical analysis was performed with the use of SPSS for Windows (SPSS Inc., Chicago, Ill., USA). Categorical data were compared by means of $\chi^{2}$ analysis and Fisher's exact test when numbers were small. Comparison of nonparametric data was done with the Mann-Whitney U test.

A logistical regression analysis was performed on the duration and level of difficulty of the LC to correct for the surgeons' experience.

A multivariate regression analysis was performed on the variables associated with outcome of bile culture.
Table 1. Patient characteristics of 64 patients with positive and negative bile cultures

\begin{tabular}{lccc}
\hline & $\begin{array}{l}\text { Bacteria } \\
\text { present } \\
(\mathrm{n}=40)\end{array}$ & $\begin{array}{l}\text { Bacteria } \\
\text { absent } \\
(\mathrm{n}=24)\end{array}$ & $\begin{array}{l}\mathrm{p} \\
\text { value }\end{array}$ \\
\hline Male/female & $18 / 22$ & $5 / 19$ & $0.053^{*}$ \\
Age, years & $60(21-85)$ & $44(24-68)$ & $<0.001$ \\
ERCP attempts & $1(1-3)$ & $1(1-3)$ & 0.290 \\
Median delay ES-LC & $38(0-533)$ & $2(0-61)$ & 0.063 \\
\hline
\end{tabular}

Data are numbers and figures in parentheses are ranges. ${ }^{*} \chi^{2}$ test.

Table 2. Multivariate logistic regression analysis on the influence of 3 variables on the outcome of bile cultures

\begin{tabular}{lllrl}
\hline Predictor & OR & $95 \% \mathrm{CI}$ & & $\mathrm{p}$ value \\
\hline Group $^{1}$ & 3.811 & 1.069 & 13.590 & 0.039 \\
Age & 1.079 & 1.031 & 1.130 & 0.001 \\
Sex & 1.946 & 0.519 & 7.304 & 0.324 \\
\hline
\end{tabular}

Data are numbers. ${ }^{1}$ ELC or DLC group.

\section{Results}

Between June 2006 and October 2008, 96 patients were included in the trial. 49 underwent LC within $72 \mathrm{~h}$ after ES (ELC) and 47 after 6-8 weeks (DLC). Of these 96 patients, 93 received the allocated intervention (96.8\%).

In 64 of the 93 included patients bile cultures were obtained (68.8\%) in 26 ELC patients (55.3\%) and 38 DLC patients (82.6\%). These 64 patients form the basis of the present study. A flowchart is given in figure 1 . There were no significant differences in this subgroup of 64 patients compared to the original group of 93 patients.

\section{Bactobilia}

The overall prevalence of bactobilia was $62.5 \%(40 / 64)$. In the ELC group, $50 \%(n=13)$ of patients had bactobilia as compared to $71.1 \%(\mathrm{n}=27)$ in the DLC group ( $\mathrm{p}=$ 0.088). Two thirds of patients $(67.5 \%)$ with bactobilia originated from the DLC group. Patient characteristics are listed in table 1. Bacteria most often cultured were Streptococcus viridans (27.5\%), Escherichia coli (25\%) and Haemophilus species (25\%). In almost $75 \%$ of the positive 
Fig. 1. Patient flowchart. LC = Laparoscopic cholecystectomy; ES = endoscopic sphincterotomy.

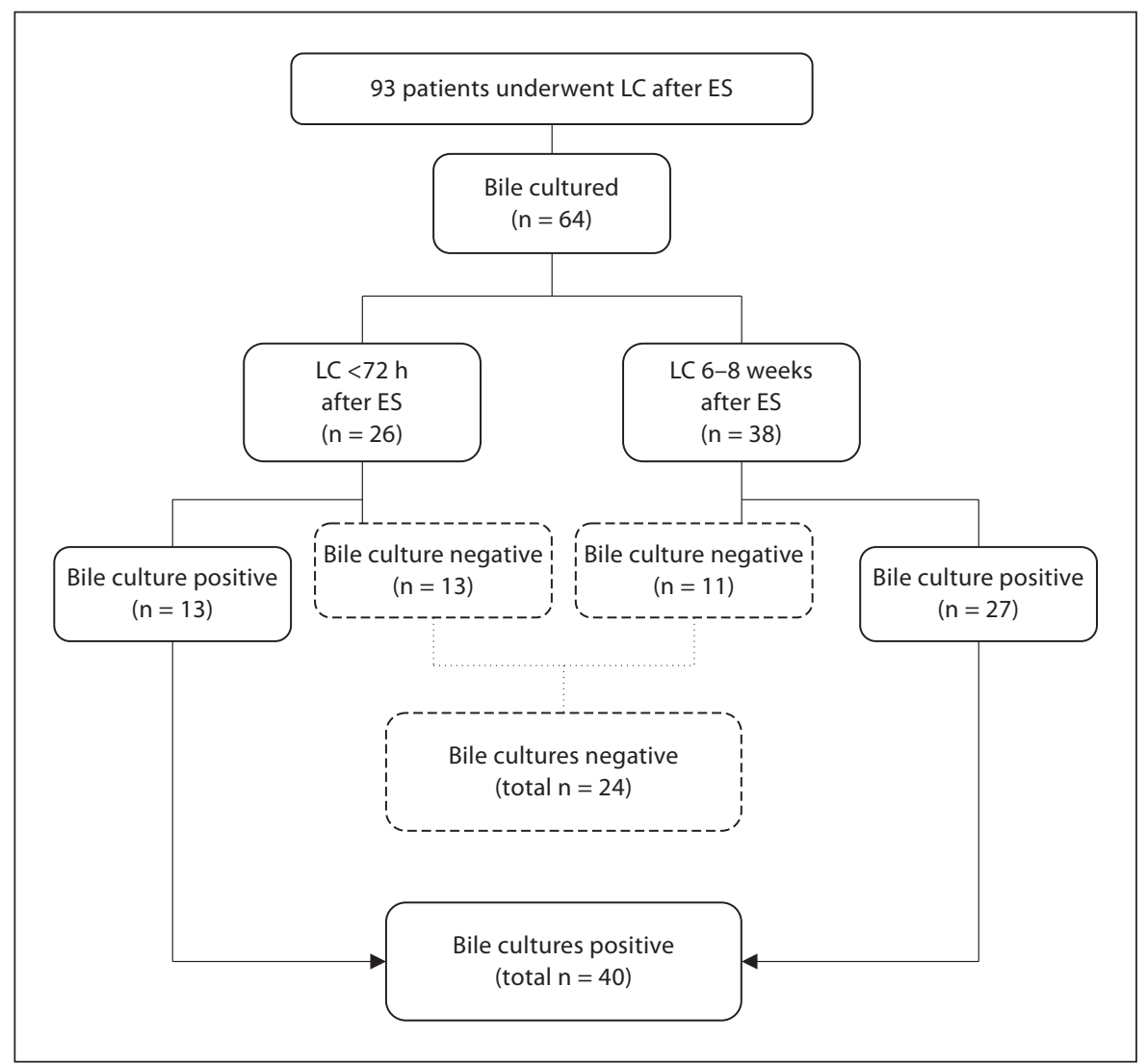

cultures multiple organisms were found, up to 4 different species per bile sample.

For the purpose of further analysis of the risk factors and surgical outcome, we divided the patients into two groups: patients with bactobilia ( $\mathrm{B}+$ group, $\mathrm{n}=40)$ and patients with sterile bile ( $\mathrm{B}$ - group, $\mathrm{n}=24)$.

Patients with bactobilia (B+ group) appeared to be significantly older than patients without bactobilia ( $\mathrm{B}$ group) $(\mathrm{p}<0.001)$ (table 1$)$. In the $\mathrm{B}+$ group, the male to female ratio is 1:1.2 compared to 1:3.8 in the B-group $(\mathrm{p}=0.053)$. The median time between ES and LC in the $\mathrm{B}+$ group was 38 days (range $0-533$ ) and for the $\mathrm{B}$ - group 2 days $(0-61)(p=0.063)$. Mean time was 48 days for the $\mathrm{B}+$ group and 21 days for the $\mathrm{B}-$ group.

Multivariate logistic regression analysis on 3 variables (group, i.e. ELC or DLC, age and sex) showed that time after ES and age were independently and significantly associated with bactobilia (table 2).

\section{Perioperative Findings}

There was no significant difference in difficulty of surgery between the $\mathrm{B}+$ and $\mathrm{B}$ - groups (table 3 ). The median operating time was $57 \mathrm{~min}$ for the $\mathrm{B}+$ group and $62 \mathrm{~min}$ for the $\mathrm{B}$ - group $(\mathrm{p}=0.884)$. There were 2 conversions in the $\mathrm{B}+$ group $(5 \%)$ compared to zero in the $\mathrm{B}$ - group $(\mathrm{p}=$ 0.524). Experience of surgeons in LC was comparable between $\mathrm{B}+$ and $\mathrm{B}-$ groups. Both conversions in the $\mathrm{B} \pm$ group were in patients originally from the DLC group.

\section{Follow-Up: Recurrent Biliary Events}

None of the patients in the original ELC group developed biliary events, very likely due to the early timing of the LC. Therefore, the recurrence of biliary events were only studied in the DLC group $(\mathrm{n}=38)$. Within that group we compared the clinical course of patients in the $\mathrm{B}+$ group $(\mathrm{n}=27)$ and patients in the $\mathrm{B}-$ group $(\mathrm{n}=11)$. In the $\mathrm{B}+$ group biliary events occurred in $44 \%$ of this subgroup of patients $(n=12)$ during follow-up. Of these 12 patients, 3 developed acute cholecystitis, 8 had biliary colics, and 1 patient developed recurrent CBD stones. All these events occurred prior to their LC (table 4). In the B- group $27 \%$ of patients $(n=3)$ suffered from biliary events, all three had biliary colics, none had acute cholecystitis $(\mathrm{p}=0.47)$. 
Table 3. Procedural characteristics of 64 patients with positive and negative bile cultures

\begin{tabular}{llll}
\hline & $\begin{array}{l}\text { Bacteria } \\
\text { present } \\
(\mathrm{n}=40)\end{array}$ & $\begin{array}{l}\text { Bacteria } \\
\text { absent } \\
(\mathrm{n}=24)\end{array}$ & $\begin{array}{l}\mathrm{p} \\
\text { value }\end{array}$ \\
\hline $\begin{array}{l}\text { LC experience of } \\
\text { surgeon, median }\end{array}$ & $>200$ & $50-200$ & 0.369 \\
$\begin{array}{l}\text { Duration of LC, min } \\
\text { Difficulty of LC }\end{array}$ & $57.5(35-120)$ & $62(30-120)$ & 0.884 \\
Conversion rate & $5(0-9)$ & $4.5(1-9)$ & 0.949 \\
\hline
\end{tabular}

Data are numbers and figures in parentheses are ranges, unless otherwise indicated.

* Fisher's exact test.

Table 4. Biliary events in patients from the DLC group $(\mathrm{n}=38)$ with positive and negative bile cultures

\begin{tabular}{llll}
\hline & $\begin{array}{l}\text { Bacteria } \\
\text { present } \\
(\mathrm{n}=27)\end{array}$ & $\begin{array}{l}\text { Bacteria } \\
\text { absent } \\
(\mathrm{n}=11)\end{array}$ & $\begin{array}{l}\mathrm{p} \\
\text { value* }\end{array}$ \\
\hline No biliary event & $15(66 \%)$ & $8(73 \%)$ & 0.47 \\
Biliary event & $12(44 \%)$ & $3(27 \%)$ & \\
$\quad$ Biliary colic & 8 & 3 & \\
Acute cholecystitis & 3 & 0 & \\
Recurrent CBD stone & 1 & 0 & \\
\hline
\end{tabular}

Data are numbers. * Fisher's exact test.

\section{Postoperative Complications}

In the $\mathrm{B}+$ group postoperative complications occurred in 3 patients $(7.5 \%$; 2 wound infections and 1 cystic duct leak, treated conservatively) (table 5). In the Bgroup 1 patient $(4.2 \%)$ suffered a complication (wound infection).

\section{Discussion}

Our study group consisted of 64 choledochocystolithiasis patients, who all underwent sphincterotomy and in most cases also mechanical removal of CBD stones. In this entire group, $62.5 \%$ had bactobilia at the time of surgery. These numbers are similar to those in a study performed by Csendes et al. [9] in which bile was aspirated in 425 patients with symptomatic gallstone disease and 42 control patients. In their control group, all bile samples
Table 5. Postoperative morbidity and hospital stay in patients with positive and negative bile cultures

\begin{tabular}{llll}
\hline & $\begin{array}{l}\text { Bacteria } \\
\text { present } \\
(\mathrm{n}=40)\end{array}$ & $\begin{array}{l}\text { Bacteria } \\
\text { absent } \\
(\mathrm{n}=24)\end{array}$ & $\begin{array}{l}\mathrm{p} \\
\text { value }\end{array}$ \\
\hline $\begin{array}{lll}\text { Complications } \\
\quad \text { Wound infection }\end{array}$ & $3(7.5 \%)$ & $1(4.2 \%)$ & $1.00^{*}$ \\
$\begin{array}{l}\text { Cystic duct leak } \\
\text { Hospital stay (range), days }\end{array}$ & 1 & 1 & \\
$\quad \begin{array}{l}\text { Postoperative } \\
\text { Total }\end{array}$ & $2(1-11)$ & $1(1-14)$ & $0.480^{* *}$ \\
& $4(2-14)$ & $4(2-16)$ & $0.198^{* *}$ \\
\hline
\end{tabular}

Data are numbers. * Fisher's exact test; ${ }^{* *}$ Mann-Whitney U test.

were sterile. In patients with uncomplicated symptomatic gallstone disease, infected bile was found in $22 \%$, and in patients with stones in the common bile duct (without cholangitis), bacteria were present in $58 \%$ of the patients. This bactobilia rate is comparable with the $50 \%$ rate we found shortly after ES. Of patients in the delayed LC group in whom the cultures were taken 6-8 weeks after sphincterotomy, $71 \%$ appeared to have bactobilia.

The cause of this increase in bactobilia over time is still unknown. It strongly suggests that destruction of the sphincter of Oddi does in fact lead to reflux, resulting in an increased colonization over time [5-9]. However, we also found bactobilia in patients receiving ES and LC the same day, making it plausible that bactobilia was present before sphincterotomy took place. This may suggest that the stones themselves are a cause of colonization and the sphincterotomy adds to this effect [10]. On the other hand, in this specific group of patients it cannot be excluded that stone passage prior to ES has itself led to mechanical dysfunction and subsequent reflux and colonization.

Bactobilia did not have any effect on the difficulty of the LC, operating time or conversion rate. Nor did patients with bactobilia have more infectious complications. This is in contrast with two other studies on the effect of bile cultures on the outcome of LC $[11,12]$. In the study by Morris-Stiff et al. [12] more infectious complications occurred in the group with positive bile cultures $(\mathrm{p}<0.05)$. Also in a study by Galili et al. [11] not only did infectious complications occur more often, but overall complications were significantly higher in the group with infected bile. These findings were not reproduced in our study, possibly due to the limited number of patients included. 
Patients with bactobilia tend to have more biliary events than those without infected bile. Three of $40 \mathrm{pa}-$ tients developed an acute cholecystitis (7.5\%) and 8 patients (20\%) suffered from biliary colics. Only 3 patients $(12.5 \%)$ in the B-group suffered biliary colics, and no acute cholecystitis was seen in this group. Even though this difference was not significant, also most likely due to limited numbers, it still shows a tendency that bactobilia might be a risk factor for developing biliary events.

Our study clearly shows an association between increasing age and bactobilia: $50 \%$ of patients with bactobilia were 60 years or older, in contrast with $12.5 \%$ of patients without infected bile $(\mathrm{p}=0.002$, likelihood ratio $=$ 10.1). This is consistent with other studies and is thought to be caused by age-dependent physiological changes (decreased biliary motility and clearance, increasing duodenal motor disorders and anatomical/functional alterations, diabetes mellitus) [9, 11-13].

Our patient population originates from a randomized clinical trial on the effects of the timing of LC after ES. Since sample size calculation of that trial was based on other primary endpoints, our study is underpowered for a number of interesting outcomes on the consequences of bactobilia (infectious and biliary complications, conversion rate). We therefore cannot provide strong recommendations on the need to perform an early LC after ES in order to prevent bactobilia from occurring, nor can we advocate prophylactic antibiotics in patients with risk factors for bactobilia (old age, increased time between ES and LC). However, it surely does stress the need for further research on this subject.

\section{Conclusions}

Almost two thirds of patients have bactobilia after ES for choledochocystolithiasis. It seems that over time bile becomes infected after ES. Delay of LC leads to a higher incidence of infected bile. In our study, bactobilia did not lead to more complications of surgery. Patients with infected bile have a tendency for developing biliary events during the waiting time for LC. It is important that more data from larger studies on this subject become available to stress the importance of early LC after ES.

\section{References}

1 McAlister V, Davenport E, Renouf E: Cholecystectomy deferral in patients with endoscopic sphincterotomy. Cochrane Database Syst Rev 2007;4:CD006233.

$\checkmark 2$ Reinders JS, Goud A, Timmer R, Kruyt PM, Witteman BJM, Smakman N, Breumelhof R, Donkervoort SC, Jansen JM, Heisterkamp J, Grubben M, van Ramshorst B, Boerma D: Early laparoscopic cholecystectomy improves outcomes after endoscopic sphincterotomy for choledochocystolithiasis. Gastroenterology 2010;138:2315-2320.

3 Salman B, Yilmaz U, Kerem M, Bedirli A, Sare M, Sakrak O, Tatlicioglu E: The timing of laparoscopic cholecystectomy after endoscopic retrograde cholangiopancreaticography in cholelithiasis coexisting with choledocholithiasis. J Hepatobiliary Pancreat Surg 2009; $16: 832-836$.

4 Sarli L, Iusco DR, Roncoroni L: Preoperative endoscopic sphincterotomy and laparoscopic cholecystectomy for the management of cholecystocholedocholithiasis: 10-year experience. World J Surg 2003;27:180-186.
-5 de Vries A, Donkervoort S, Geloven van AA, Pierik EG: Conversion rate of laparoscopic cholecystectomy after endoscopic retrograde cholangiography in the treatment of choledocholithiasis: does the time interval matter? Surg Endosc 2005; 19:996-1001.

-6 Sung JY, Leung JW, Shaffer EA, Lam K, Olson ME, Costerton JW: Ascending infection of the biliary tract after surgical sphincterotomy and biliary stenting. J Gastroenterol Hepatol 1992;7:240-245.

7 Sand J, Airo I, Hiltunen KM, Mattila J, Nordback I: Changes in biliary bacteria after endoscopic cholangiography and sphincterotomy. Am Surg 1992;58:324-328.

8 Mandryka Y, Klimczak J, Duszewski M, Kondras M, Modzelewski B: Bile duct infections as a late complication after endoscopic sphincterotomy. Pol Merkur Lekarski 2006; 21:525-527. $\checkmark 9$ Csendes A, Burdiles P, Maluenda F, Diaz JC, Csendes P, Mitru N: Simultaneous assessment of bile from gallbladder and common bile duct in control subjects and patients with gallstones and common duct stones. Arch Surg 1996;131:389-394.

10 Maurer KJ, Carey MC, Fox JG: Roles of infection, inflammation, and the immune system in cholesterol gallstone formation. Gastroenterology 2009;138:425-440.

-11 Galili O, Eldar Jr S, Matter I, Madi H, Brodsky A, Galis I, Eldar Sr S: The effect of bactibilia on the course and outcome of laparoscopic cholecystectomy. Eur J Clin Microbiol Infect Dis 2008;27:797-803.

12 Morris-Stiff GJ, O’Donohue P, Ogunbiyi S, Sheridan WG: Microbiological assessment of bile during cholecystectomy: is all bile infected? HPB 2007;9:225-228.

13 Hickman MS, Schwesinger WH, Page CP: Acute cholecystitis in the diabetic. Arch Surg 1988;123:409-411. 